\title{
Tuberculoid leprosy masquerading as systemic lupus erythematosus: an interesting observation
}

\author{
Vijay Zawar ${ }^{1}$, Shrikant Kumavat ${ }^{\circledR}{ }^{凶}$ Manoj Pawar $^{2}$, Dipti Desai ${ }^{3}$
}

\begin{abstract}
Leprosy is a chronic granulomatous infectious multisystem disease that may present with protean manifestations. It mimics many systemic and dermatological disorders. Here we report a case in which an elderly female presented with malar rash, intermittent fever, and arthralgia. Her diagnosis was significantly delayed due to a close clinical resemblance to systemic lupus erythematosus. It is important to be aware of such manifestations of leprosy and improve awareness of it in clinicians to avoid misdiagnosis and delay in treatment.
\end{abstract}

Keywords: leprosy, lupus, mimic

Received: 28 March 2017| Returned for modification: 15 June 2017 | Accepted: 30 June 2017

\section{Introduction}

Leprosy is an infectious disease caused by Mycobacterium leprae. It primarily affects the skin and peripheral nerves, but also other parts of the body such as the bones, liver, eyes, reticuloendothelial system, vascular endothelium, muscles, and upper respiratory tract (1). Leprosy may be clinically confused with other systemic diseases such as systemic lupus erythematosus (SLE), rheumatoid arthritis, sarcoidosis, drug rash, granuloma annulare, granuloma multiforme, pityriasis rotunda, erythema multiforme, and many others (2).

\section{Case report}

A 58-year-old female patient was referred by a physician to us for consultation on persistent malar rash for 6 months. Earlier, she had consulted him for intermittent low-grade fever, pain in the small joints of the hands and legs, fatigue, and anorexia of 1 year duration. There was mild photosensitivity over the malar areas. She was not taking any drugs before the onset of the symptoms and was healthy in the past. There was no family or past history of leprosy. She had traveled few times in the previous 4 years to relatives living in areas with higher endemicity for leprosy. Her complete blood counts, random blood sugar level, serum uric acid, liver function tests, renal function tests, and rheumatoid factor were within normal limits except for ESR, which was $80 \mathrm{~mm}$. HIV and VDRL tests were negative. Her antinuclear antibody (ANA) by ELISA was positive with titer 1:120, but double stranded DNA (ds-DNA) was negative. Her chest X-ray and serum angiotensin-converting enzyme level were normal. She was diagnosed as a case of dsDNA-negative SLE and was initially prescribed analgesics with hydroxychloroquine. However, due to inadequate response she was switched over to oral prednisolone $30 \mathrm{mg}$ per day and tapered gradually. Her other symptoms partially responded, but the malar rash persisted despite 6 months of this treatment. Hence she was referred to us.

Upon dermatological examination, she had well-defined erythematous plaques over both malar regions with slight scaling involving part of the right malar area and upper lip, sparing the nasolabial fold (Fig. 1). A non-scaly bright erythematous plaque was also noted over the left submental area (Fig. 2). There was hypoesthesia to light touch and temperature over the malar and submental plaques. Her left ulnar and both lateral popliteal nerves were slightly thickened and tender. The palmoplantar skin and mucosal surfaces were not affected, and there was no lymphadenopathy.

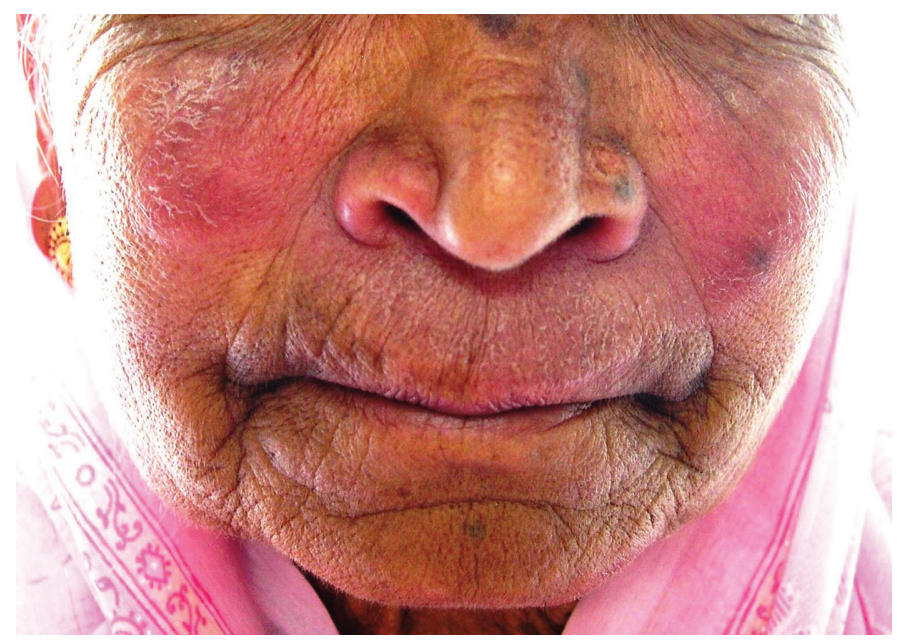

Figure 1 | Erythematous edematous well-defined plaques over both malar regions with scaling on the right malar area and part of the upper lip.

A potassium hydroxide wet preparation from the skin eruption was negative for fungus. Slit skin smears were negative for acid-fast bacilli. A skin biopsy was taken from the plaque on the malar region, which revealed typical tuberculoid granulomas in the upper dermis consisting of epithelioid cells, Langhans giant cells, and dense lymphocytic infiltrate with dermal edema suggestive of tuberculoid leprosy with mild type-one reaction (Fig. 3). Thus, a diagnosis of tuberculoid leprosy was made and it took about 1.5 years from the onset of symptoms to a concrete diagnosis. Daily oral dapsone $100 \mathrm{mg}$ and rifampicin $600 \mathrm{mg}$ once a month for 6 months, and systemic prednisolone in tapering doses with intermittent aceclofenac were prescribed to the patient. This treatment led to complete resolution of the rash and all of her systemic symptoms within 6 months. Her malar plaques gradually flattened and later completely resolved (Fig. 4). She was followed for the next year and she did not require any further therapy. 


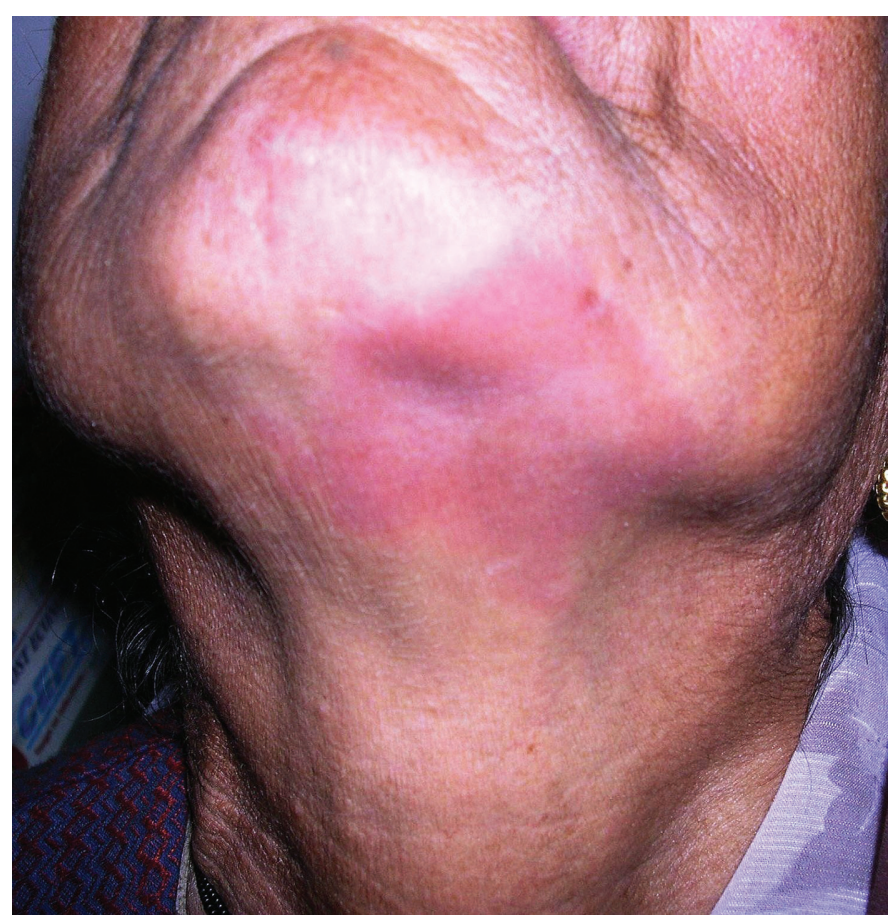

Figure $2 \mid \mathrm{A}$ bright red erythematous well-defined plaque over the left submental area.

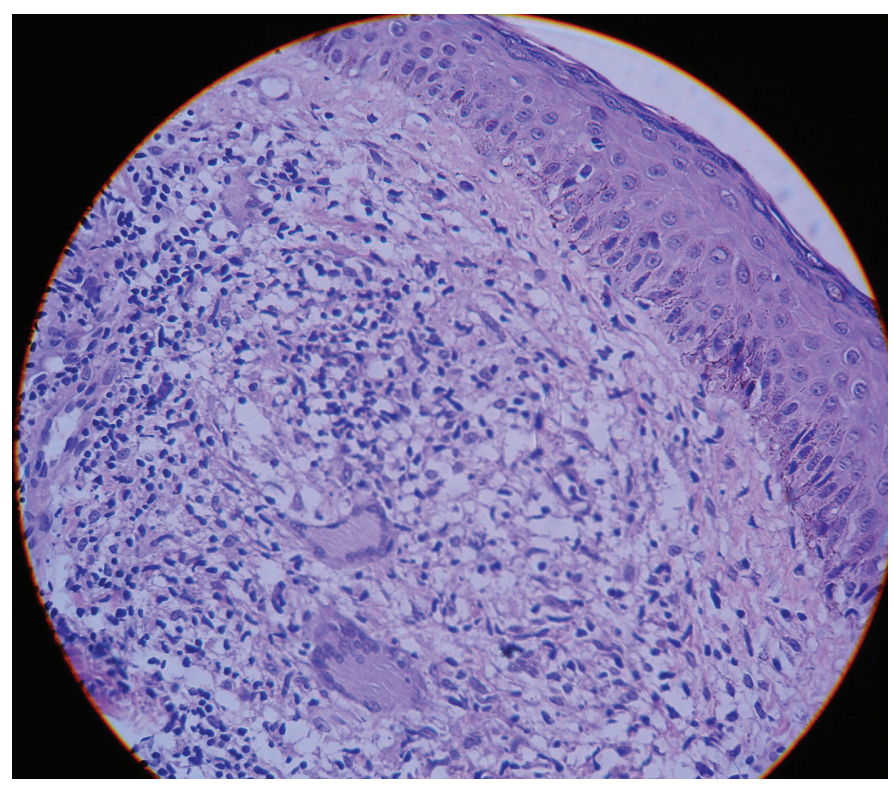

Figure 3 | Histopathology showing thickened epidermis. The dermis shows a granulomatous infiltration consisting of lymphocytes surrounding Langhans giant cells, epithelioid cells, and perivascular dense lymphocytic infiltrate with edema $(H \& E \times 40)$.

\section{Discussion}

Cutaneous manifestations of leprosy are diverse and can be hypopigmented macules, annular plaques, or nodules. Tuberculoid leprosy presents with macules or plaques. Plaques are generally one to three in number and vary from $0.5 \mathrm{~cm}$ to $30 \mathrm{~cm}$ in size. It affects the face, extremities, trunk, and buttocks, and is usually asymmetrical. The surface of the plaques may be dry and slightly scaly. Sensations over these plaques are characteristically impaired.

SLE is one of the connective tissue diseases involving various systems in the body with a myriad of clinical presentations. SLE is characterized by butterfly-shaped erythematous plaques on the face with photosensitivity. Arthralgia and arthritis involving the symmetric small joints of the extremities and occasionally the 82

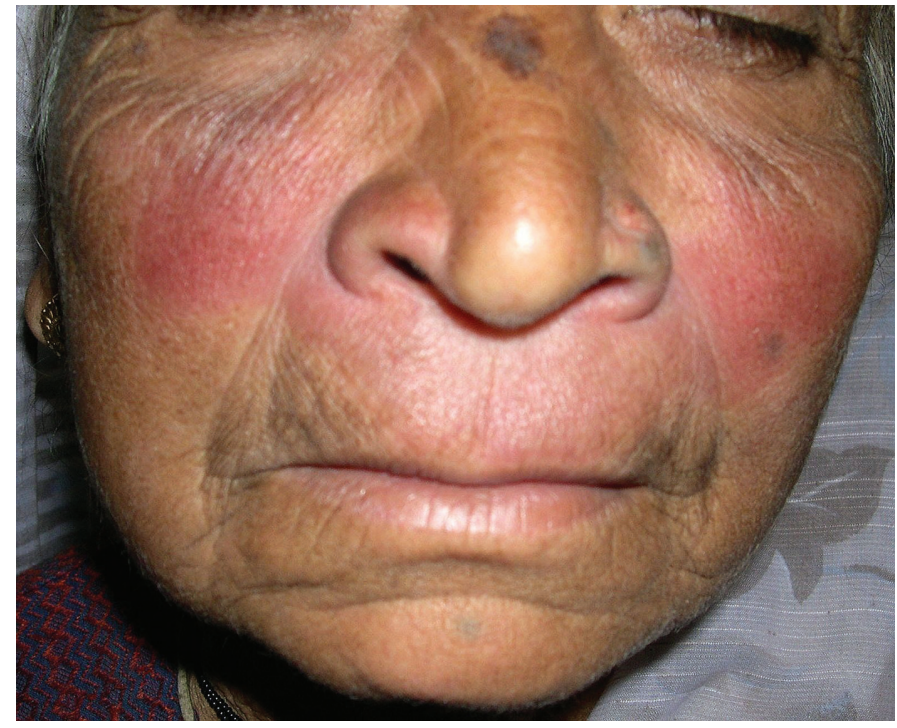

Figure 4 | Malar plaques flattened after 6 months of multidrug therapy for leprosy.

elbows and knees are common extracutaneous features. Antinuclear antibodies (a mostly homogeneous pattern) and anti-dsDNA antibodies are present in the serum of SLE patients (3).

Leprosy closely mimics SLE because of similarities in their cutaneous, rheumatic, neurologic, and serologic features, and both diseases can also coexist (4-6). In our patient, the rash over the malar region resembled the butterfly rash of SLE, but there was hypoesthesia upon examination. Although hypoesthesia or anesthesia of the skin lesion is an important diagnostic criterion of leprosy, it may be absent or there may be only minimal anesthesia over the face because of the rich overlapping nerve supply. Peripheral neuropathy, which is quite evident in leprosy patients, can also be observed in certain SLE patients, the prevalence of which varies from 5 to $27 \%$ (7). However, the neuropathy of SLE has a predilection for a younger age group and the lower limbs, especially the peroneal and sural nerves, and it also has a shorter duration. Polyneuropathy of SLE mostly occurs in patients with a high SLE disease activity index and those with central nervous system involvement. In addition to skin and peripheral nervous system involvement, osteoarticular involvement is one of the most common manifestations of leprosy (8). The proximal interphalangeal joints, metacarpophalangeal joints, elbows, and knees are mainly involved in reactional states of leprosy, which are also commonly involved in SLE, adding a further diagnostic dilemma. Antinuclear antibodies are reported in lepromatous leprosy and lepra reactions, varying from 3 to $34 \%$ but in low titers $(9,10)$.

Auto-aggressive Hansen's disease is a rare presentation of lepromatous leprosy or borderline leprosy, which shares clinical features of connective tissue diseases such as SLE (11).

Our patient lives in a village $40 \mathrm{~km}$ from Nashik, the district capital. The annual prevalence rate of leprosy is 1.21 in Nashik. Thus, the area is not hyperendemic for leprosy as such (12). She may have contracted the infection from a highly endemic area that she traveled to a few years previously.

The clinical progress in our patient was initially rheumatologic manifestations (intermittent low-grade fever, pain in the small joints of the hands and legs, fatigue, and anorexia) followed by malar rash. Persistent non-responsive erythematous plaque and thickened peripheral nerves prompted us to consider leprosy in our patient. Complete resolution of the cutaneous lesions following a course of anti-leprosy treatment confirmed the diagnosis of leprosy. 
Because this patient fulfilled four major criteria put forth by the American College of Rheumatology Revised Criteria, she was diagnosed with SLE. However, one should reconsider the criteria in the setting of endemic areas for leprosy (13).

\section{Conclusion}

Timely diagnosis and appropriate treatment of leprosy is impor-

\section{References}

1. Hastings RC. Leprosy. 2nd ed. New York: Churchill Livingstone; c1994. Chapter 6, Pathology of leprosy; p. 193-24.

2. Hastings RC. Leprosy. 2nd ed. New York: Churchill Livingstone; C1994. Chapter 8, Differential diagnosis; p. 291-16.

3. Burns T, Breathnach S, Cox N, Griffiths C. Rook's textbook of dermatology. 8th ed. Oxford: Wiley-Blackwell; c2010. Chapter 51, The connective tissue diseases; p. 51.1-51.64.

4. Zorbas P, Kontochristopoulos G, Detsi I, Apostolakis E, Kouris N, Katsamhas A. Borderline tuberculoid leprosy coexisting with systemic lupus erythematosus. J Eur Acad Dermatol Venereol. 1999;12:274-5.

5. Ohkawa S, Ozaki M, Izumi S. Lepromatous leprosy complicated with systemic lupus erythematosus. Dermatologica. 1985;170:80-3.

6. Posner DI, Guill MA. Coexistent leprosy and lupus erythematosus. Cutis. 1987;39:136-8.

7. Garg G, Gogia A, Kakar A. Leprosy: a great mimicking disease. Indian J Lepr. 2016; 88:123-7. tant, especially in geographical areas where leprosy is a public health threat. Our case also illustrates the importance of careful detailed clinical examination and follow-up. Clinicians must be aware of presentations of leprosy that mimic SLE.

8. Pereira HL, Ribeiro SL, Pennini SN, Sato El. Leprosy-related joint involvement. Clin Rheumatol. 2009;28:79-84.

9. Chavez-Legaspi M, Gomez-Vazquez A, García-De La Torre I. Study of rheumatic manifestations and serologic abnormalities in patients with lepromatous leprosy. J Rheumatol. 1985;12:738-41.

10. Guedes BLS, Gilbrut B, Shoenfeld Y, Scheinderg MA. Autoantibodies in leprosy sera. Clin Rheumatol. 1996;15:26-8.

11. Dhar S, Kanwar AJ, Kaur S. Autoaggressive lepromatous leprosy. Int J Lepr. 1993;61:467-8.

12. nlep.nic.in: District wise PR (prevalence rate) \& NCDR (national case detection rate) as on March 2015 [Internet]. [cited 2017 Jun 16]. Available from: http:// nlep.nic.in/pdf/Disttwise\%20NCDR-Mar.15\%20.pdf.

13. Teixeira Junior GJ, Silva CE, Magalhães V. Application of the diagnostic criteria for systemic lupus erythematosus to patients with multibacillary leprosy. Rev Soc Bras Med Trop. 2011;44:85-90. 